\title{
Normalized Histogram of the State Variable of First-order Digital Filters with Two's Complement Arithmetic
}

\author{
Bingo Wing-Kuen Ling ${ }^{1}$, Charlotte Yuk-Fan $\mathrm{Ho}^{2}$ and Peter Kwong-Shun Tam ${ }^{3}$ \\ ${ }^{I}$ Department of Electronic Engineering, Division of Engineering, King's College London, Strand, \\ London, WC2R 2LS, United Kingdom. \\ ${ }^{2}$ Department of Electronic Engineering, Queen Mary, University of London, Mile End Road, London, E1 \\ 4NS, United Kingdom. \\ ${ }^{3}$ Department of Electronic and Information Engineering, The Hong Kong Polytechnic University, Hung \\ Hom, Kowloon, Hong Kong, PRC.
}

\begin{abstract}
In this letter, the normalized histogram of the state variable of first-order digital filters with two's complement arithmetic is investigated. When the pole of the digital filter is between 1 and 2 , it is found that the possibility of occurrence of the state variable in certain region is close to zero no matter what the initial condition is. Some analytic results are given to account for this phenomenon.
\end{abstract}

Keywords—Digital filters, two's complement arithmetic, normalized histogram.

\section{INTRODUCTION}

The two's complement arithmetic is commonly used for the implementation of addition and subtraction operations in digital filters. However, overflow may occur. As a consequence, digital filters with two's complement arithmetic may exhibit limit cycle 
behaviors [1] or chaotic behaviors [2]-[11].

The existing investigations on the chaotic behaviors of digital filters with two's complement arithmetic are mainly on the admissibility and periodicity of the symbolic sequences [2], [4], [6]-[11]. Some investigations have been extended to the saturation-type [3] and quantization-type [5] nonlinearities. However, very little literature has discussed on the statistical properties of the state variables of these systems [12]. When the digital filters exhibit chaotic behaviors, one may expect that the state variables may reach any values between the maximum and minimum numbers in the possible region and uniform probability distribution may obtain. The condition for obtaining a uniform distribution was derived in [12]. However, we do not know the distribution when the condition stated in [12] violates. In this letter, we find that the possibility of occurrence of the state variable of first-order digital filters with two's complement arithmetic is close to zero in certain region no matter what the initial condition is. In this letter, we investigate this issue and some analytical results are given.

An outline for the other sections of this letter is as follows: Analytical and simulation results are presented in Section II. Conclusion is given in Section III.

\section{ANALYTICAL AND SIMULATION RESULTS}

Assume that a first-order digital filter with two's complement arithmetic can be represented by the following state space model:

$$
x(k+1)=f(a \cdot x(k)+u(k)),
$$

where $a$ is the filter parameter, $u(k)$ is the input signal, $x(k)$ is the state variable, and $f$ is the nonlinearity due to the use of two's complement arithmetic, which is the Frobenius-Perron operator [12]. In this letter, we assume that $u(k)=0$ for $k \geq 0$ and the whole system is only influenced by the initial state. The nonlinearity $f$ can be 
modeled as:

$$
f(v)=v-2 \cdot n
$$

such that

$$
2 \cdot n-1 \leq v<2 \cdot n+1
$$

and $n \in Z$, in which $Z$ denotes the set of integers. Hence, the digital filters with two's complement arithmetic can be represented as:

$$
x(k+1)=a \cdot x(k)+2 \cdot s(k), \text { for } k \geq 0,
$$

where $-1 \leq x(k)<1$ and $s(k) \in\{-m, \cdots,-1,0,1, \cdots, m\}$, in which $m$ is the minimum integer satisfying $-2 \cdot m-1 \leq a \cdot x(k)<2 \cdot m+1$. Since $s(k)$ is an element in the discrete set $\{-m, \cdots,-1,0,1, \cdots, m\}$, the values of $s(k)$ can be viewed as symbols and $s(k)$ is called a symbolic sequence.

In this letter, we only consider the case when $1<a<2$. In this case, since the pole of the digital filter is outside the unit circle, random like chaotic behaviors would occur and it is expected that in the long run the state variable may reach any values in $[-1,1)$. However, we have conducted extensive simulations and find that the possibility of occurrence of the state variable in certain region is close to zero. The results are summarized in the following Lemma.

\section{Lemma 1:}

If $1<a<2$, the possibility of occurrence of the state variable in the region $(a-2,2-a)$ is close to zero.

\section{Proof}

Although the digital filters with two's complement arithmetic are deterministic systems, that is, the values of the state variable and the symbolic sequence at any particular time index can be calculated exactly once the initial condition and the filter parameter are known, we can always plot the histogram of the state variable and compute the possibility of occurrence of different values of the symbolic sequences. 
Let the normalized histogram of the state variable be $p_{X}(x)$, that is $\int_{-1}^{1} p_{X}(x) d x=1$.

Define

$$
\begin{gathered}
q(x)=\left\{\begin{array}{cc}
p_{X}(x) & -\frac{1}{a} \leq x<\frac{1}{a}, \\
0 & \text { otherwise }
\end{array}\right. \\
r(x)=\left\{\begin{array}{cc}
p_{X}(x) & \frac{1}{a} \leq x<1 \\
0 & \text { otherwise }
\end{array}\right. \\
t(x)=\left\{\begin{array}{cc}
p_{X}(x) & -1 \leq x<-\frac{1}{a} \\
0 & \text { otherwise }
\end{array}\right. \\
y=f(a \cdot x),
\end{gathered}
$$

and the normalized histogram of $y$ be $p_{Y}(y)$, where $\int_{-1}^{1} p_{Y}(y) d y=1$, then we have:

$$
p_{X}(x)=q(x)+r(x)+t(x)
$$

and

$$
p_{Y}(x)=\frac{1}{a} \cdot q\left(\frac{x}{a}\right)+\frac{1}{a} \cdot r\left(\frac{x+2}{a}\right)+\frac{1}{a} \cdot t\left(\frac{x-2}{a}\right) .
$$

For the digital filters with two's complement arithmetic, we can assume $p_{Y}(x)=p_{X}(x)$. Hence we have:

$$
\begin{aligned}
t(x)= & \frac{1}{a} \cdot q\left(\frac{x}{a}\right)+\frac{1}{a} \cdot r\left(\frac{x+2}{a}\right), \text { for }-1 \leq x<-\frac{1}{a}, \\
q(x)= & \frac{1}{a} \cdot q\left(\frac{x}{a}\right)+\frac{1}{a} \cdot r\left(\frac{x+2}{a}\right), \text { for }-\frac{1}{a} \leq x<a-2, \\
& q(x)=\frac{1}{a} \cdot q\left(\frac{x}{a}\right), \text { for } a-2 \leq x<2-a, \\
q(x)= & \frac{1}{a} \cdot q\left(\frac{x}{a}\right)+\frac{1}{a} \cdot t\left(\frac{x-2}{a}\right), \text { for } 2-a \leq x<\frac{1}{a},
\end{aligned}
$$

and

$$
r(x)=\frac{1}{a} \cdot q\left(\frac{x}{a}\right)+\frac{1}{a} \cdot t\left(\frac{x-2}{a}\right), \text { for } \frac{1}{a} \leq x<1 .
$$

According to (13), we have $q(x)=\frac{c}{|x|}$ where $c$ is a constant, or $q(x)=0$. Since 
$q(x)=\frac{c}{|x|}$ is not a valid normalized histogram function because $\int_{a-2}^{2-a} q(x) d x$ diverges, so we have $q(x)=0$. This implies that the possibility of occurrence of the state variable in the region $(a-2,2-a)$ is close to zero.

Figure 1 shows the simulation results when $a=1.5$ and $x(0)=0.8$. It can be seen from Figure 1a that although the digital filter with two's complement arithmetic exhibits random like chaotic behaviors, the possibility of occurrence of the state variable in the region $(a-2,2-a)$ is close to zero, as shown in Figure 1 b.
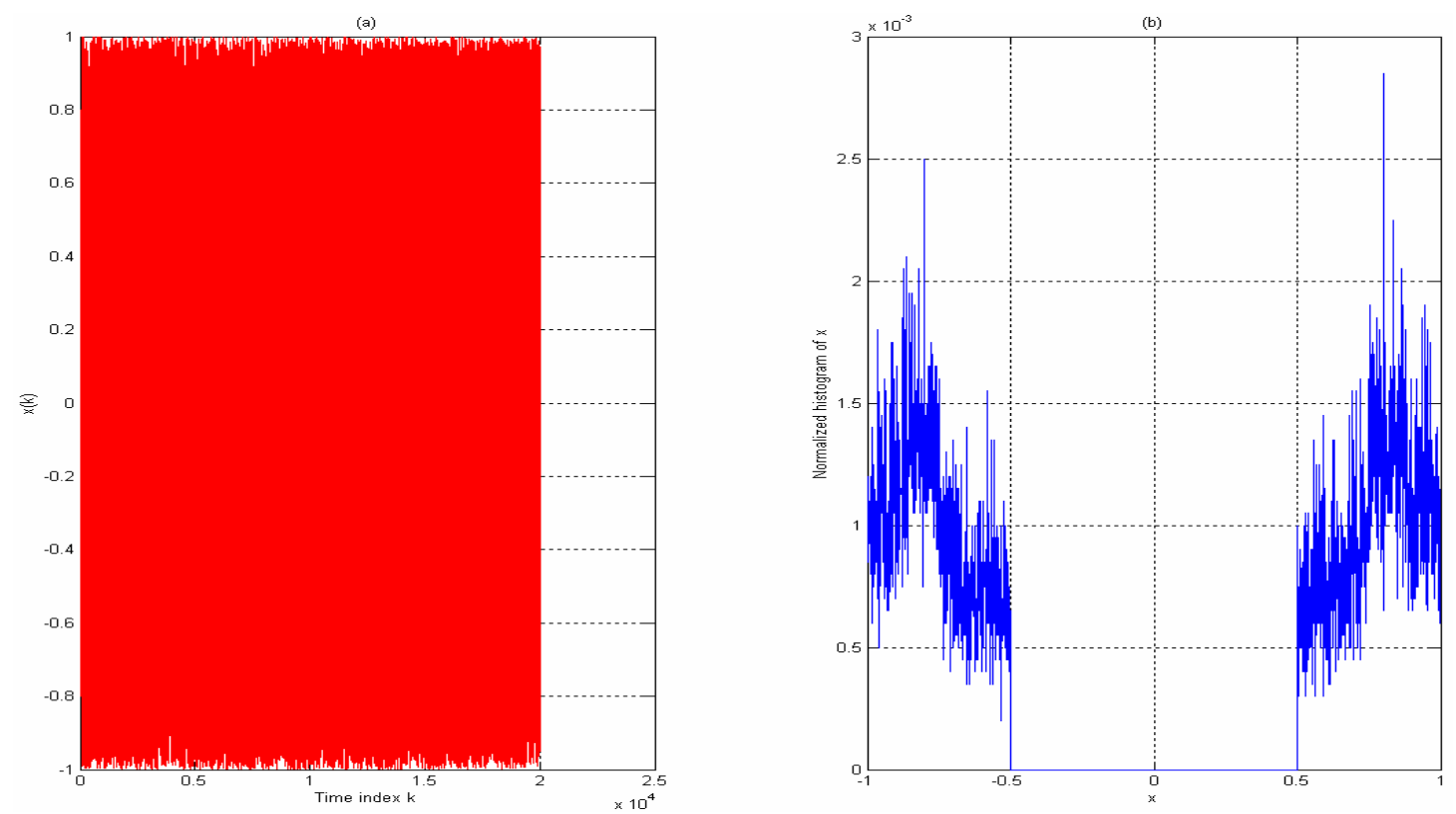

Figure 1. (a) $x(k)$. (b) The normalized histogram of the state variable when $a=1.5$ and $x(0)=0.8$.

It is worth noting that even though the state trajectory depends on the initial condition, the derivation of Lemma 1 does not involve the initial condition. So, our results are still valid no matter what the initial conditions are. Figure 2 shows the simulation results when $a=1.5$ and $x(0)$ is generated randomly. We can see that Figure 2 depicts results which are similar to those of Figure 1. 

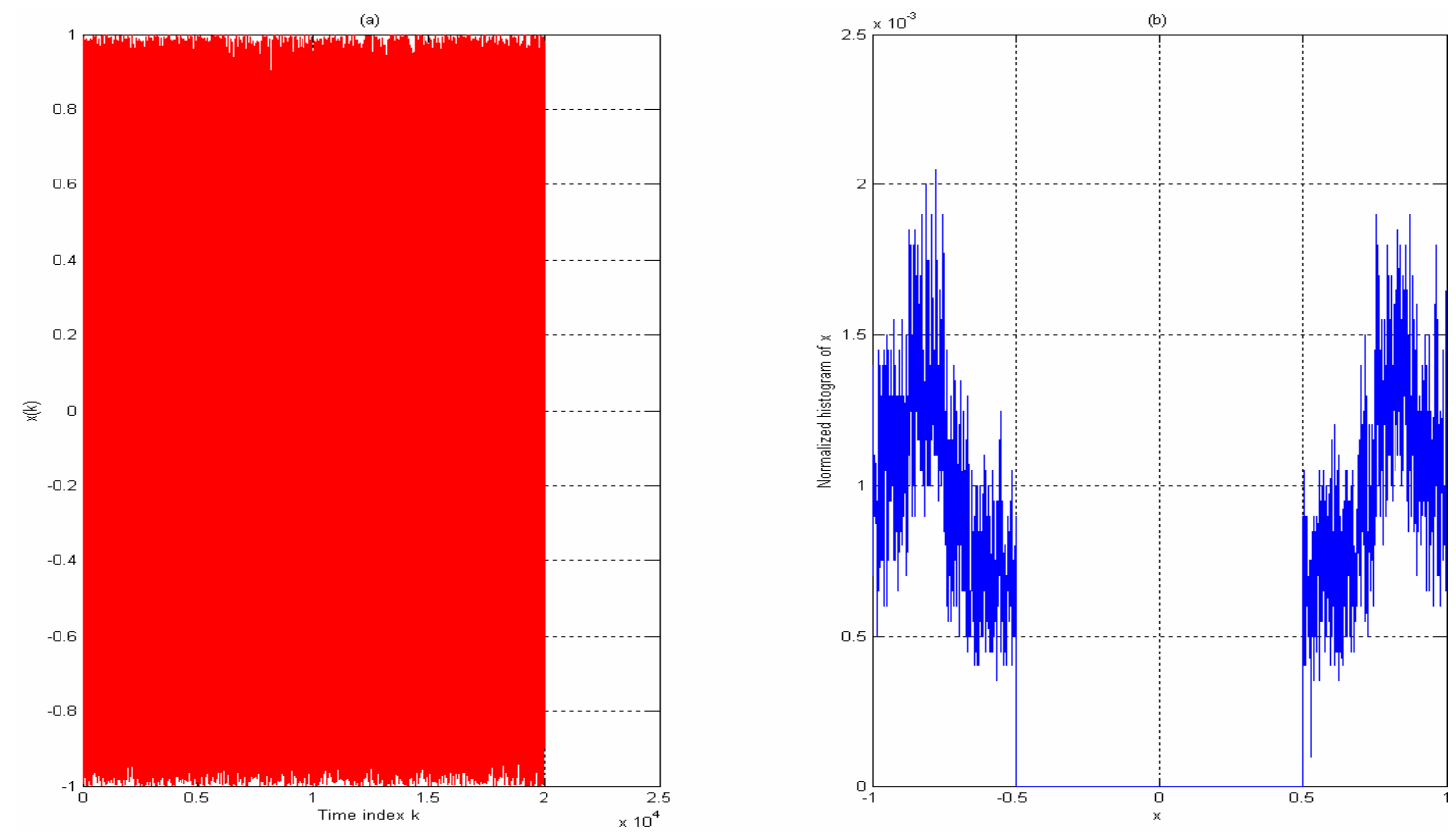

Figure 2. (a) $x(k)$. (b) The normalized histogram of the state variable when $a=1.5$ and the initial condition is generated randomly.

\section{CONCLUSION}

In this letter, we have investigated the normalized histogram of the state variable of first-order digital filters with two's complement arithmetic. When the pole of the digital filters with two's complement arithmetic is between 1 and 2, it is found and explained that the possibility of occurrence of the state variable in certain region is very close to zero.

\section{ACKNOWLEDGEMENT}

The work obtained in this letter was supported by a research grant (project number G-YD26) from the Hong Kong Polytechnic University.

\section{REFERENCES}

[1] G. F. Xu and T. Bose, "Elimination of limit cycles due to two's complement 
quantization in normal form digital filters," IEEE Transactions on Signal Processing, vol. 45, no. 12, pp. 2891-2895, 1997.

[2] L. O. Chua and T. Lin, "Chaos in digital filters," IEEE Transactions on Circuits and Systems, vol. 35, no. 6, pp. 648-658, 1988.

[3] Z. Galias and M. J. Ogorzalek, "Bifurcation phenomena in second-order digital filter with saturation-type adder overflow characteristic," IEEE Transactions on Circuits and Systems, vol. 37, no. 8, pp. 1068-1070, 1990.

[4] L. O. Chua and T. Lin, "Fractal pattern of second-order non-linear digital filters: a new symbolic analysis," International Journal of Circuit Theory and Applications, vol. 18 , pp. 541-550, 1990.

[5] T. Lin and L. O. Chua, "On chaos of digital filters in the real world," IEEE Transactions on Circuits and Systems, vol. 38, no. 5, pp. 557-558, 1991.

[6] Z. Galias and M. J. Ogorzalek, "On symbolic dynamics of a chaotic second-order digital filter," International Journal of Circuit Theory and Applications, vol. 20, pp. 401-409, 1992.

[7] C. W. Wu and L. O. Chua, "Properties of admissible symbolic sequences in a second-order digital filter with overflow non-linearity," International Journal of Circuit Theory and Applications, vol. 21, pp. 299-307, 1993.

[8] L. Kocarev and L. O. Chua, "On chaos in digital filters: case $b=-1$," IEEE Transactions on Circuits and Systems-II: Analog and Digital Signal Processing, vol. 40, no. 6, pp. 404-407, 1993.

[9] L. Kocarev, C. W. Wu and L. O. Chua, "Complex behavior in digital filters with overflow nonlinearity: analytical results," IEEE Transactions on Circuits and Systems-II: Analog and Digital Signal Processing, vol. 43, no. 3, pp. 234-246, 1996.

[10]X. Yu and Z. Galias, "Periodic behaviors in a digital filter with two's complement 
arithmetic," IEEE Transactions on Circuits and Systems-I: Fundamental Theory and Applications, vol. 48, no. 10, pp. 1177-1190, 2001.

[11]L. O. Chua and T. Lin, "Chaos and fractals from third-order digital filters," International Journal of Circuit Theory and Applications, vol. 18, pp. 241-255, 1990.

[12]K. Kelber, "N-dimensional uniform probability distribution in nonlinear autoregressive filter structures," IEEE Transactions on Circuits and Systems-I: Fundamental Theory and Applications, vol. 47, no. 9, pp. 1413-1417, 2000. 